Nota de investigación

\title{
Características de calidad de la masa de harina integral y refinada y la relación con volumen de pan
}

\author{
Daniel Hernández Clemente ${ }^{1}$ \\ Eliel Martínez Cruz ${ }^{2 \S}$ \\ Azalia Lozano Grande ${ }^{1}$ \\ Víctor Morales Guzmán ${ }^{1}$ \\ Ernesto Solís Moya ${ }^{3}$ \\ Rene Hortelano Santa Rosa ${ }^{2}$ \\ ${ }^{1}$ Universidad Tecnológica de Xicotepec de Juárez Puebla. (reyes.dh34@gmail.com; \\ mariaazalia.lozano@utxicotepec.edu.mx; victor.morales@utxicotepec.edu.mx). ${ }^{2}$ Campo Experimental \\ Valle de México-INIFAP. (hortelano.rene@inifap.gob.mx). ${ }^{3}$ Campo Experimental Bajío-INIFAP. \\ (solis.ernesto@inifap.gob.mx). \\ ${ }^{\S}$ Autor para correspondencia: martinez.eliel@inifap.gob.mx.
}

\section{Resumen}

Dentro del programa de mejoramiento genético de trigo harinero la calidad panadera como criterio de selección es tardado y costoso por lo que se deben identificar variables que ayuden a seleccionarla indirectamente. El objetivo de esta investigación fue relacionar las variables de amasado en harina integral con las características industriales de las masas y su volumen de pan en harina refinada. Se midió el contenido de proteína y volumen de sedimentación en harina refinada, tiempo y estabilidad al amasado, tolerancia sobre el amasado y altura del mixógrama en harina integral y refinada, fuerza de la masa, relación tenacidad-extensibilidad y volumen de pan en harina refinada. Se obtuvo el valor promedio y las correlaciones de Pearson, se clasificaron los genotipos con base en su volumen de pan y se realizó una comparación de medias. El volumen de pan se correlacionó positivamente con el tiempo de amasado en harina integral (0.6). Además, la estabilidad al amasado en harina integral se correlacionó con tiempo de amasado en harina refinada (0.5) y fuerza de la masa (0.5). Los volúmenes de pan superiores a $800 \mathrm{cc}$ presentaron tiempos de amasado de 2.4 a 2.9 min en harina integral. Por lo que el uso del tiempo y estabilidad del amasado en harina integral ayudaría a eficientar la selección de genotipos con masas de características panificables.

Palabras clave: Triticum aestivum L., características de amasado, harina integral, volumen de pan.

Recibido: febrero de 2021

Aceptado: marzo de 2021 
Uno de los criterios de selección más importantes dentro del programa de mejoramiento de trigo harinero (Triticum aestivum L.) del Instituto Nacional de Investigaciones Forestales Agrícolas y Pecuarias (INIFAP) es la selección de líneas con mayor calidad panadera (Espitia-Rangel et al., 2017). Dicha calidad panadera se mide como volumen de pan lo cual comercialmente se estima como mayor número de piezas de pan por cantidad de harina utilizada. La determinación de dicho volumen se realiza en una hogaza originada de $100 \mathrm{~g}$ de harina refinada por lo que se requieren al menos $200 \mathrm{~g}$ de grano para obtener la cantidad de harina indicada así mismo el ensayo requiere de aproximadamente $4 \mathrm{~h}$ para su realización, además del uso de equipo especializado como el fermentador y horno eléctrico.

Por lo que, debido al tamaño de muestra utilizado, tiempo empleado para realizar la prueba y uso de infraestructura especializada es importante identificar variables de la masa que ayuden indirectamente a seleccionar líneas con mayor volumen de pan utilizando menor cantidad de grano, tiempo y equipo. En este contexto diversos autores han indicado correlaciones significativas del volumen de pan con variables determinadas en harina refinada como son proteína en harina (Dowell et al., 1989), volumen de sedimentación, (Takata et al., 1999) tiempo de amasado en el mixografo (Miles et al., 2020) así como, fuerza de la masa y la relación entre tenacidadextensibilidad determinadas en el alveógrafo (Guzmán et al., 2017). Sin embargo, falta estudiar en mayor medida la relación del volumen de pan con las propiedades de amasado en harina integral. El objetivo de la investigación fue relacionar las características de amasado mediante el mixógrafo utilizando harina integral con las variables mixográficas, alveográficas y de calidad panadera determinadas en harina refinada.

Se utilizaron 39 líneas del programa de mejoramiento de trigo harinero del Campo Experimental Bajío (CEBAJ). Para la obtención de la harina integral se molieron $50 \mathrm{~g}$ de grano sin adición de agua en un molino tipo UDY utilizando una malla de $0.5 \mathrm{~mm}$ posteriormente se tamizaron las muestras con una malla $8 \mathrm{xx}$ por dos minutos para obtener una harina con tamaño de partícula homogénea. Para la obtención de la harina refinada la molienda se realizó en un molino Bhuler (Brabender OHG, Alemania) según el método 26-31 de la AACC (2005). En la harina refinada se determinó el contenido de proteína (\%) con el analizador de reflectancia de infrarrojo cercano (NIR Feed \& Forage 5000) utilizando el método 39-00 de la AACC (2005).

Asimismo, con la harina refinada se obtuvo mediante la prueba de Zeleny el volumen de sedimentación (ml) (método 56-61A; AACC, 2005) con 3.2 g de harina en presencia de azul de bromofenol y alcohol isopropilico. El tiempo de amasado (min), tolerancia al sobreamasado (mm), estabilidad al amasado (min) y altura del mixógrama $(\mathrm{mm})$, se estimó mediante el mixógrafo (Nacional Manufacturing Co., Lincoln, NE) usando el método 54-40A de la AACC (2005) en muestras de $10 \mathrm{~g}$ de harina integral y refinada. La fuerza (W) y la relación de tenacidad/extensibilidad (PL) de la masa, se calcularon del alveógrama el cual se obtuvo de $60 \mathrm{~g}$ de harina refinada utilizando el alveógrafo de Chopin (Tripette \& Renaud, Francia) usando el método 54-30A de la AACC (2005).

El volumen de pan $(\mathrm{ml})$ se realizó mediante el método de masa directa (método 10-09, AACC, 2005) a partir de $100 \mathrm{~g}$ de harina refinada y se determinó en un volutómetro por desplazamiento de semillas de colza (Brassica campestris L.). Se obtuvo el valor promedio, desviación estándar y rango para cada una de las variables analizadas además se realizaron correlaciones mediante la 
prueba de Pearson entre variables. Adicionalmente apartir de los grupos generados con base en su volumen de pan, grupo 1 mayores a $900 \mathrm{cc}$, grupo 2 de 800 a $900 \mathrm{cc}$, grupo 3 de 700 a $800 \mathrm{cc}$ y grupo 4 de 600 a $700 \mathrm{cc}$, se realizó la prueba de Tukey $(\alpha=0.05)$ para identificar las diferencias.

En el Cuadro 1 se presentan los valores promedio, máximos y mínimos para cada una de las variables evaluadas. Los valores promedio para tiempo de amasado en harina integral y refinada fueron similares (2.5 y 2.6 min, respectivamente) y sus rangos fueron de 1.6 a 3.5 y de 1.3 a 3.9 min, respectivamente. Con base al alveógrama la fuerza promedio de la masa fue de 252 y varió de masa débil $\left(84 \times 10^{-4} \mathrm{~J}\right)$ a masa fuerte $\left(524 \times 10^{-4} \mathrm{~J}\right)$. La relación tenacidad-extensibilidad (PL) tuvo un promedio de 1.1 y rangos de 0.2 a 4.3. Por lo que con base a las variables de fuerza existen masas en los genotipos analizados como débiles $\left(\mathrm{W}<200 \times 10^{-4} \mathrm{~J}\right)$, medias $\left(200\right.$ a $\left.300 \times 10^{-4} \mathrm{~J}\right) \mathrm{y}$ fuertes $\left(>300 \times 10^{-4} \mathrm{~J}\right)$.

Cuadro 1. Media, valores mínimos y máximos y desviación estándar (DS) de calidad industrial de genotipos de trigo harinero.

\begin{tabular}{ccccc}
\hline Variable & Media & Mínimo & Máximo & DS \\
\hline Tiempo de amasado en harina integral $(\mathrm{min})$ & 2.5 & 1.6 & 3.5 & 0.5 \\
Tolerancia al sobreamasado en harina integral $(\mathrm{mm})$ & 10 & 3 & 14 & 2.6 \\
Estabilidad al amasado en harina integral $(\mathrm{min})$ & 3.4 & 2.3 & 5.3 & 0.7 \\
Altura del mixógrama en harina integral $(\mathrm{mm})$ & 74.9 & 62 & 91 & 6.8 \\
Proteína en harina refinada $(\%)$ & 10.3 & 9.3 & 11.9 & 0.6 \\
Volumen de sedimentación $(\mathrm{ml})$ & 29.1 & 20 & 38 & 4.7 \\
Tiempo de amasado en harina refinada $(\mathrm{min})$ & 2.6 & 1.3 & 3.9 & 0.6 \\
Tolerancia al sobreamasado en harina refinada $(\mathrm{mm})$ & 4.9 & 1 & 10 & 2.3 \\
Estabilidad al amasado en harina refinada $(\mathrm{min})$ & 4.1 & 2.1 & 5.6 & 1 \\
Altura del mixógrama en harina refinada $(\mathrm{mm})$ & 62.2 & 52 & 72 & 5.5 \\
Fuerza de la masa (x10 $\left.{ }^{-4} \mathrm{~J}\right)$ & 252 & 84 & 524 & 109.5 \\
Relación tenacidad-extensibilidad & 1.1 & 0.2 & 4.3 & 0.8 \\
Volumen de pan $(\mathrm{cc})$ & 804.6 & 620 & 1010 & 108.2 \\
\hline
\end{tabular}

Mientras que por su relación tenacidad-extensibilidad se clasificaron como extensibles por su $\mathrm{P} / \mathrm{L}<$ 1, balanceadas por su $\mathrm{PL}=1$ y tenaces por su $\mathrm{P} / \mathrm{L}>1.2$. Por las características de la masa, anteriormente señaladas, el volumen de pan tuvo un promedio de $804.6 \mathrm{cc}$ y un rango desde 620 a 1010 cc lo cual indica que en el grupo de genotipos existen líneas de trigo con volumen de pan clasificado como regular de 500 a $600 \mathrm{cc}$, hasta excelentes $>900 \mathrm{cc}$.

En el Cuadro 2 se presentan las correlaciones de Pearson entre las variables analizadas. El parámetro importante que define la calidad panadera es el volumen de pan el cual se correlacionó alto y positivamente con volumen de sedimentación (0.7), tiempo de amasado en harina integral (0.6), fuerza de la masa (0.6) y tiempo de amasado en harina refinada (0.5), Cuadro 2. Resultados similares en harina refinada fueron encontrados para tiempo de amasado en el mixógrafo por Baasandorj et al. (2020); asimismo, Villaseñor et al. (2017) indicaron que mayor fuerza asociada a extensibilidad de la masa, determinada en el alveógrafo, favorecen el volumen del pan. 
Cuadro 2. Correlaciones de Pearson entre variables de calidad industrial de genotipos de trigo harinero.

\begin{tabular}{|c|c|c|c|c|c|c|c|c|c|c|c|c|}
\hline & TSAHI & EAMHI & AMHI & $\mathrm{PH}$ & VS & TAHR & TSAHR & EMHR & AMHR & W & PL & VP \\
\hline TAHI & $-0.4^{*}$ & $0.4^{* *}$ & $0.1 \mathrm{~ns}$ & $0.01 \mathrm{~ns}$ & $0.7^{* *}$ & $0.8^{* *}$ & $-0.1 \mathrm{~ns}$ & $0.3^{*}$ & $0.08 \mathrm{~ns}$ & $0.7^{* *}$ & $0.02 \mathrm{~ns}$ & $0.6^{* *}$ \\
\hline TSAHI & & $-0.7^{* *}$ & $0.3 \mathrm{~ns}$ & $0.1 \mathrm{~ns}$ & $-0.3^{*}$ & $-0.4^{*}$ & $0.03 \mathrm{~ns}$ & $-0.2 \mathrm{~ns}$ & $-0.01 n s$ & $-0.5^{* *}$ & $-0.1 \mathrm{~ns}$ & $-0.2 \mathrm{~ns}$ \\
\hline EAMHI & & & $0.1 \mathrm{~ns}$ & $-0.1 \mathrm{~ns}$ & $0.4^{*}$ & $0.5^{* *}$ & $-0.3 n s$ & $0.4^{*}$ & $0.1 \mathrm{~ns}$ & $0.5^{* * *}$ & $0.03 \mathrm{~ns}$ & $0.2 \mathrm{~ns}$ \\
\hline AMHI & & & & $0.5^{* *}$ & $0.3^{*}$ & $0.2 \mathrm{~ns}$ & $-0.2 \mathrm{~ns}$ & $0.3^{*}$ & $0.1 \mathrm{~ns}$ & $0.2 \mathrm{~ns}$ & $0.1 \mathrm{~ns}$ & $-0.01 \mathrm{~ns}$ \\
\hline $\mathrm{PH}$ & & & & & $0.1 \mathrm{~ns}$ & $0.01 \mathrm{~ns}$ & $-0.1 \mathrm{~ns}$ & $0.1 \mathrm{~ns}$ & $-0.07 n s$ & $0.2 \mathrm{~ns}$ & $-0.2 \mathrm{~ns}$ & $0.03 \mathrm{~ns}$ \\
\hline VS & & & & & & $0.7^{* *}$ & $-0.3 n s$ & $0.4^{*}$ & $0.1 \mathrm{~ns}$ & $0.7^{* * *}$ & $-0.2 \mathrm{~ns}$ & $0.7^{* *}$ \\
\hline TAHR & & & & & & & $-0.3 \mathrm{~ns}$ & $0.4^{*}$ & $0.1 \mathrm{~ns}$ & $0.7^{* * *}$ & $0.04 \mathrm{~ns}$ & $0.5^{* *}$ \\
\hline TSAHR & & & & & & & & $-0.8^{* *}$ & $0.4^{*}$ & $-0.4^{*}$ & $0.2 \mathrm{~ns}$ & $-0.3^{*}$ \\
\hline EAMHR & & & & & & & & & $-0.2 \mathrm{~ns}$ & $0.5^{* *}$ & $-0.05 \mathrm{~ns}$ & $0.3^{*}$ \\
\hline AMHR & & & & & & & & & & $0.01 \mathrm{~ns}$ & $0.3^{*}$ & $-0.1 \mathrm{~ns}$ \\
\hline W & & & & & & & & & & & $-0.01 \mathrm{~ns}$ & $0.6^{* *}$ \\
\hline PL & & & & & & & & & & & & $-0.5^{* *}$ \\
\hline
\end{tabular}

TAHI; TSAHI y EAMHI= tiempo, tolerancia, estabilidad al amasado en harina integral, respectivamente; AMHI= altura del mixógrama en harina integral; $\mathrm{PH}=$ proteína en harina; $\mathrm{VS}=$ volumen de sedimentación; TAHR; TSAHR y EAMHR= tiempo, tolerancia y estabilidad al amasado en harina refinada, respectivamente; AMHR= altura del mixograma en harina refinada; $\mathrm{W}=$ fuerza de la masa; $\mathrm{PL}=$ relación tenacidad-extensibilidad; $\mathrm{V}=$ volumen de pan.

Por otro lado, también se encontraron correlaciones altas y significativas del tiempo de amasado en harina integral con tiempo de amasado en harina refinada (0.8), volumen de sedimentación (0.7) y fuerza de la masa (0.7). Por lo que el tiempo de amasado en harina integral es un variable que nos puede ayudar a seleccionar para mayor fuerza de la masa y volumen de pan, al igual que el tiempo de amasado y volumen de sedimentación en harina refinada.

Así mismo la estabilidad al amasado en harina integral se correlacionó positivamente con tiempo de amasado en harina refinada (0.5) y fuerza de la masa (0.5). De tal modo que el tiempo y estabilidad al amasado en harina integral son adecuadas para seleccionar genotipos con mayor fuerza de la masa y consecuentemente favorecer el volumen de pan.

En el Cuadro 3 se presentan los promedios de las variables estudiadas por grupo de calidad con base a su volumen de pan. El grupo 1 de mayor volumen de pan clasificado como excelente por su valor superior a 900 cc presentó tiempos de amasado de 2.9 y 3.1 min en harina integral y refinada, respectivamente. Así como volumen de sedimentación de $33 \mathrm{ml}$ en harina refinada. Mientras que para el grupo 2 de volumen de pan catalogado como muy bueno de 800 a 900 cc mostró valores de tiempo de amasado de 2.4 y 2.5 min para harina integral y refinada, respectivamente. Con base a sus variables alveográficas el grupo 1 se caracterizó por tener masas fuertes $\left(\mathrm{W}=331.1 \times 10^{-4} \mathrm{~J}\right)$ asociado a masas extensibles por su relación tenacidad- extensibilidad (PL) menor a 1. 
Cuadro 3. Comparación de medias de las variables de calidad industrial por grupo (G) de calidad panadera.

\begin{tabular}{cccccccccccccc}
\hline G & \multicolumn{2}{c}{$\begin{array}{c}\text { TAHI TSAHI EAMHI } \\
(\mathrm{min})\end{array}$} & $\begin{array}{c}\text { AMHI } \\
(\mathrm{mm})\end{array}$ & $\begin{array}{c}\text { PH } \\
(\mathrm{min})\end{array}$ & $\begin{array}{c}\text { VS } \\
(\mathrm{mm})\end{array}$ & $\begin{array}{c}\text { TAHR TSAHR EAMHR AMHR } \\
(\%)\end{array}$ & $\begin{array}{c}\mathrm{W} \\
(\mathrm{ml})\end{array}$ & $\begin{array}{c}\mathrm{W} \text { (min) } \\
(\mathrm{mm})\end{array}$ & $\begin{array}{c}\text { PL } \\
(\mathrm{min})\end{array}$ & $\begin{array}{c}\text { VP } \\
(\mathrm{mm})\end{array}$ & $\left(\mathrm{x} 10^{-4} \mathrm{~J}\right)$ & $(0.1-6)$ & $(\mathrm{cc})$ \\
\hline 1 & $2.9 \mathrm{a}$ & $9.2 \mathrm{a}$ & $3.5 \mathrm{a}$ & $75.4 \mathrm{a}$ & $10.3 \mathrm{a}$ & $33.3 \mathrm{a}$ & $3.1 \mathrm{a}$ & $4.1 \mathrm{a}$ & $4.5 \mathrm{a}$ & $61.5 \mathrm{a}$ & $331.1 \mathrm{a}$ & $0.7 \mathrm{~b}$ & $929 \mathrm{a}$ \\
2 & $2.4 \mathrm{~b}$ & $9.4 \mathrm{a}$ & $3.7 \mathrm{a}$ & $74.2 \mathrm{a}$ & $10.3 \mathrm{a}$ & $30 \mathrm{ba}$ & $2.5 \mathrm{ba}$ & $4.2 \mathrm{a}$ & $4.4 \mathrm{a}$ & $62.5 \mathrm{a}$ & $291.6 \mathrm{a}$ & $0.9 \mathrm{~b}$ & $848.1 \mathrm{~b}$ \\
3 & $2.2 \mathrm{~b}$ & $11 \mathrm{a}$ & $3.2 \mathrm{a}$ & $73.6 \mathrm{a}$ & $10 \mathrm{a}$ & $25.2 \mathrm{c}$ & $2.3 \mathrm{~b}$ & $5.4 \mathrm{a}$ & $3.7 \mathrm{a}$ & $60.1 \mathrm{a}$ & $169.5 \mathrm{~b}$ & $1 \mathrm{~b}$ & $727.2 \mathrm{c}$ \\
4 & $2.1 \mathrm{~b}$ & $10.7 \mathrm{a}$ & $3.1 \mathrm{a}$ & $76.2 \mathrm{a}$ & $10.4 \mathrm{a}$ & $26.1 \mathrm{bc}$ & $2.3 \mathrm{~b}$ & $6.3 \mathrm{a}$ & $3.6 \mathrm{a}$ & $65.1 \mathrm{a}$ & $181.3 \mathrm{~b}$ & $2 \mathrm{a}$ & $660.5 \mathrm{~d}$ \\
\hline
\end{tabular}

Grupo 1=> $900 \mathrm{cc}$, grupo 2 de 800 a $900 \mathrm{cc}$, grupo 3 de 700 a $800 \mathrm{cc}$, grupo 4 de 600 a $700 \mathrm{cc}$, TAHI, TSAHI y EAMHI= tiempo, tolerancia, estabilidad al amasado en harina integral, respectivamente; $\mathrm{AMHI}=$ altura del mixógrama en harina integral; $\mathrm{PH}=$ proteína en harina; VS= volumen de sedimentación; TAHR, TSAHR y EAMHR= tiempo, tolerancia y estabilidad al amasado en harina refinada, respectivamente; AMHR= altura del mixógrama en harina refinada; $\mathrm{W}=$ fuerza de la masa; $\mathrm{PL}=$ relación tenacidad-extensibilidad; $\mathrm{VP}=$ volumen de pan.

El grupo 2 presentó masas medias y fuertes, por sus valores de $\mathrm{W}=291.6$ y por su PL se clasificó como extensibles. Por otro lado, los grupos 3 y 4 con volúmenes de pan menores a $800 \mathrm{cc}$ presentaron tiempos de amasado menores a 2.3 en harina integral y 2.4 en harina refinada. Por otro lado, presentaron valores de fuerza de la masa menores a $200 \times 10^{-4} \mathrm{~J}$ que los clasifica como masas débiles. El grupo cuatro presentó valores altos de tenacidad $\mathrm{PL}=2$, lo cual se reflejó en su valor más bajo de volumen de pan.

\section{Conclusiones}

Con base a lo anterior, las variables tiempo y estabilidad al amasado determinadas en $10 \mathrm{~g}$ de harina integral mediante el mixógrafo, pueden utilizarse para seleccionar genotipos que favorezcan la fuerza de la masa e indirectamente el volumen de pan. Lo anterior ayudará a discriminar en menor tiempo y tamaño de muestra, genotipos con poca fuerza y menor calidad panadera.

\section{Literatura citada}

AACC. 2005. American Association of Cereal Chemists. Approved Methods of the AACC. $10^{\text {th }}$ (Ed.). American Association of Cereal Chemists. St. Paul, MN, USA.

Baasandorj, T.; Ohm, J. B. and Simsek, S. 2020. Comparison of different experimental breadmaking methods and their associations with flour quality parameters in hard red spring wheat. Cereal Chem. 97(2):515-526.

Dowell, F. E.; Maghirang, E. B.; Pierce, R. O.; Lookhart, G. L.; Bean, S. R.; Xie, F.; Caley, M. S.; Wilson, J. D.; Seabourn, B. W.; Ram, M. S.; Park, S. H. and Chung O. K 2008. Relationship of bread quality to kernel, flour, and dough properties. Cereal Chem. 85(1):82-91.

Espitia-Rangel, E.; Villaseñor-Mir, H. E.; Martínez-Cruz, E.; Santa-Rosa, R. H.; Lozano-Grande, A. y Limón-Ortega, A. 2017. Análisis de la estabilidad del volumen del pan de trigos harineros (Triticum aestivum L.) mexicanos de secano. Agrociencia. 51(7):743-754.

Guzmán, C.; Autrique, E.; Mondal, S.; Huerta-Espino, J.; Singh, R. P.; Vargas, M.; Crossa, J.; Amaya, A. and Peña, R. J. 2017. Genetic improvement of grain quality traits for CIMMYT semi-dwarf spring bread wheat varieties developed during 1965-2015:50 years of breeding. Field Crops Res. 210(1):192-196. 
Miles, C.; Booyse, M.; van Biljon, A. and Labuschagne, M. 2020. The effect of different milling procedures on dough mixing parameters of hard red bread wheat. Cereal Res. Comm. 48(4):477-483.

Takata, K.; Yamauchi, H. and Kuwabara, T. 1999. Prediction of bread-making quality by prolonged swelling SDS-sedimentation test. Breeding Sci.49(3):221-223.

Villaseñor, M. H. E.; Martínez, C. E.; Hortelano, S. R. R; González, G. M.; Zamudio, C. A.; Huerta, E. J. y Espitia, R. E. 2017. Variabilidad genética y criterios de selección para calidad industrial de trigos introducidos en condiciones de temporal. Rev. Mex. Cien. Agríc. 8(3):661-672. 